\title{
Protecting-group-free synthesis of carboxylic group substituted alkynes by Sonogashira coupling in water
}

\author{
Xin-Yi Zhu, Yang Wang, Fang Bai, Guo-Wei Gao, and Jian Men* \\ College of Chemistry, Sichuan University, Chengdu, China, 610064 \\ E-mail: menjian@scu.edu.cn
}

\begin{abstract}
An effective, economic and green synthesis of carboxylic group substituted alkynes by Sonogashira coupling of halogenated aromatic acids with phenylacetylene has been developed. The coupling reaction proceeds well using $\mathrm{Pd}(\mathrm{OAc})_{2} / \mathrm{TPPTS}-\mathrm{CuI}$ as catalyst with $\mathrm{K}_{2} \mathrm{CO}_{3}$ as base in water. It is worth noting that this procedure avoids the protection/deprotection steps of carboxylic group and obtains the title compounds in good to excellent yields.
\end{abstract}

Keywords: Halogenated aromatic acids, Sonogashira coupling, phenylacetylene, water, palladium acetate

\section{Introduction}

Carboxylic group substituted alkynes have found important applications in natural products, bioactive compounds and advanced materials. ${ }^{1-3}$ In addition, substituted alkynes bearing carboxylic group are useful intermediates which can be reduced for the preparation of some simplified hybrid inhibitors, ${ }^{4}$ and also may act as trapping sites to the metal ions. ${ }^{5}$ In the traditional strategy, these compounds were synthesized by the esterification of halogenated aromatic acids, followed by Sonogashira coupling between the halogenated aromatic carboxylic esters and terminal alkynes and then subsequently hydrolysis of the coupling products to release the carboxylic group. ${ }^{6-8}$ As we know, protecting group introduction and removal has led to many drawbacks such as loss of materials, unsatisfactory yields, cumbersome experimental and formation of toxic byproducts. ${ }^{9}$

Recently, our group has reported an effective procedure for synthesis of 4-phenylethynylphthalic anhydride from 4-bromophthalic acid reacted with phenylacetylene by Sonogashira coupling reaction using $\mathrm{Pd}\left(\mathrm{PPh}_{3}\right) \mathrm{Cl}_{2}-\mathrm{CuI} / \mathrm{PPh}_{3}$ system in $\mathrm{THF} / \mathrm{Et} \mathrm{N}_{3},{ }^{10}$ which avoided the additional protection/deprotection steps of carboxylic group. And to the best of our knowledge, only a few publications have appeared employing the direct coupling between 
halogenated aromatic acids and terminal alkynes. ${ }^{11,12}$ However, in all of the above catalytic proceses, organic solvents were used as the reaction media.

Given the increasing concern about green and safe chemical processes, the application of water instead of organic solvents as the reaction media has led to a tremendous interest in Sonogashira coupling. ${ }^{13-22}$ Compared with organic solvents, water is inexpensive, nonflammable, nontoxic, and environmental friendly. Therefore, considering the above subjects and aiming at a simple and environmentally benign synthesis of carboxylic group substituted alkynes, we have investigated the direct coupling reaction of phenylacetylene with a range of halogenated aromatic acids catalyzed by available water-soluble palladium complexes in aqueous medium. Herein, we report an effective, one-step and green Sonogashira coupling for the production of a series of substituted alkynes bearing carboxylic group in water.

\section{Results and Discussion}

For the optimization of the reaction conditions, we chose the coupling of 4-bromobenzoic acid with phenylacetylene as the model reaction using $\mathrm{Pd}(\mathrm{OAc})_{2}$ as catalyst, and the effect of various reaction parameters was examined (Table 1). As shown in Table 1, the reaction was significantly influenced by cocatalyst. Without CuI, the yield dropped from 78 to $21 \%$ (Table 1, entry 1 vs. 2). It indicated that the role of $\mathrm{CuI}$ was important to facilitate the reaction. Considering that phosphine ligand plays an important role in Sonogashira coupling reactions, ${ }^{23}$ the effect of m-trisulfonated triphenylphosphine (TPPTS) on this reaction was investigated, and the results suggested that the use of TPPTS as ligand provided an efficient catalytic system (Table 1, entry 2 vs. 3). Besides, the yield increased slightly when a phase-transfer agent such as tetrabutylammonium bromide (TBAB) was added (Table 1, entry 2 vs. 4), indicating that addition of TBAB enhanced the reaction by transferring phenylacetylene to aqueous phase. The influences of different bases were also screened. It was clear that the reaction proceeded well when inorganic bases, such as $\mathrm{NaOH}, \mathrm{Na}_{2} \mathrm{CO}_{3}, \mathrm{~K}_{2} \mathrm{CO}_{3}$ and $\mathrm{Cs}_{2} \mathrm{CO}_{3}$ were used (Table 1, entries 4-7), and the best result was obtained in the case of $\mathrm{K}_{2} \mathrm{CO}_{3}$ as the base (Table 1, entry 4). However, organic base $\left(\mathrm{Et}_{3} \mathrm{~N}\right)$ gave an inferior result under the same conditions (Table 1, entry 8). Finally, reaction time and temperatures were evaluated (Table 1, entries 4, 11, 12 and 4, 9, 10). The results showed that the reaction time of $12 \mathrm{~h}$ at $100{ }^{\circ} \mathrm{C}$ gave a highest yield of $82 \%$ (Table 1, entry 4). 
Table 1. Experimental conditions for the coupling of 4-bromobenzoic acid with phenylacetylene in water ${ }^{\mathrm{a}}$

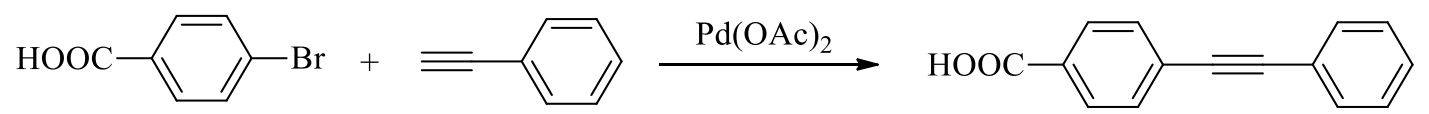

\begin{tabular}{ccccccc}
\hline Entry & Catalyst system & Additive & Base & $\begin{array}{l}\text { Temperature } \\
\left({ }^{\circ} \mathrm{C}\right)\end{array}$ & Time (h) & Yield (\%) ${ }^{\mathrm{b}}$ \\
\hline 1 & $\mathrm{Pd}(\mathrm{OAc})_{2}, \mathrm{TPPTS}$ & & $\mathrm{K}_{2} \mathrm{CO}_{3}$ & 100 & 12 & 21 \\
2 & $\mathrm{Pd}(\mathrm{OAc})_{2}, \mathrm{TPPTS}, \mathrm{CuI}$ & & $\mathrm{K}_{2} \mathrm{CO}_{3}$ & 100 & 12 & 78 \\
3 & $\mathrm{Pd}(\mathrm{OAc})_{2}, \mathrm{CuI}$ & & $\mathrm{K}_{2} \mathrm{CO}_{3}$ & 100 & 12 & 41 \\
4 & $\mathrm{Pd}(\mathrm{OAc})_{2}, \mathrm{TPPTS}, \mathrm{CuI}$ & $\mathrm{TBAB}$ & $\mathrm{K}_{2} \mathrm{CO}_{3}$ & 100 & 12 & 82 \\
5 & $\mathrm{Pd}(\mathrm{OAc})_{2}, \mathrm{TPPTS}, \mathrm{CuI}$ & $\mathrm{TBAB}$ & $\mathrm{NaOH}_{2}$ & 100 & 12 & 54 \\
6 & $\mathrm{Pd}(\mathrm{OAc})_{2}, \mathrm{TPPTS}, \mathrm{CuI}$ & $\mathrm{TBAB}$ & $\mathrm{Na}_{2} \mathrm{CO}_{3}$ & 100 & 12 & 69 \\
7 & $\mathrm{Pd}(\mathrm{OAc})_{2}, \mathrm{TPPTS}, \mathrm{CuI}$ & $\mathrm{TBAB}$ & $\mathrm{Cs}_{2} \mathrm{CO}_{3}$ & 100 & 12 & 77 \\
8 & $\mathrm{Pd}(\mathrm{OAc})_{2}, \mathrm{TPPTS}, \mathrm{CuI}$ & $\mathrm{TBAB}$ & $\mathrm{Et}_{3} \mathrm{~N}_{2}$ & 100 & 12 & 9 \\
9 & $\mathrm{Pd}(\mathrm{OAc})_{2}, \mathrm{TPPTS}, \mathrm{CuI}$ & $\mathrm{TBAB}$ & $\mathrm{K}_{2} \mathrm{CO}_{3}$ & 80 & 12 & 70 \\
10 & $\mathrm{Pd}(\mathrm{OAc})_{2}, \mathrm{TPPTS}, \mathrm{CuI}$ & $\mathrm{TBAB}$ & $\mathrm{K}_{2} \mathrm{CO}_{3}$ & 60 & 12 & 32 \\
11 & $\mathrm{Pd}(\mathrm{OAc})_{2}, \mathrm{TPPTS}, \mathrm{CuI}$ & $\mathrm{TBAB}$ & $\mathrm{K}_{2} \mathrm{CO}_{3}$ & 100 & 16 & 82 \\
12 & $\mathrm{Pd}(\mathrm{OAc})_{2}, \mathrm{TPPTS}, \mathrm{CuI}$ & $\mathrm{TBAB}$ & $\mathrm{K}_{2} \mathrm{CO}_{3}$ & 100 & 8 & 76 \\
\hline
\end{tabular}

${ }^{a}$ Reaction conditions: 4-bromobenzoic acid (10 mmol), phenylacetylene $(15 \mathrm{mmol}), \mathrm{Pd}(\mathrm{OAc})_{2}(2$ mol\%), TPPTS (8 mol\%), CuI (2 mol\%), TBAB (6 mol\%), base (20 mmol) and $\mathrm{H}_{2} \mathrm{O}(20 \mathrm{ml})$.

${ }^{\mathrm{b}}$ Isolated yield.

Having optimized the reaction conditions of phenylacetylene with 4-bromobenzoic acid, we designed and carried out some new Sonogashira coupling of halogenated aromatic acids with phenylacetylene in water (Table 2). All the reactions were completed under the above conditions except 3-hydroxy-5-iodobenzoic acid and 3-hydroxy-5-bromobenzoic acid, and the amount of $\mathrm{K}_{2} \mathrm{CO}_{3}$ was increased as their hydroxyl group would also react with $\mathrm{K}_{2} \mathrm{CO}_{3}$ (Table 2, entry 5 and $6)$. 
Table 2. Synthesis of carboxylic group substituted alkynes by Sonogashira coupling of halogenated aromatic acids with phenylacetylene in water ${ }^{\mathrm{a}}$

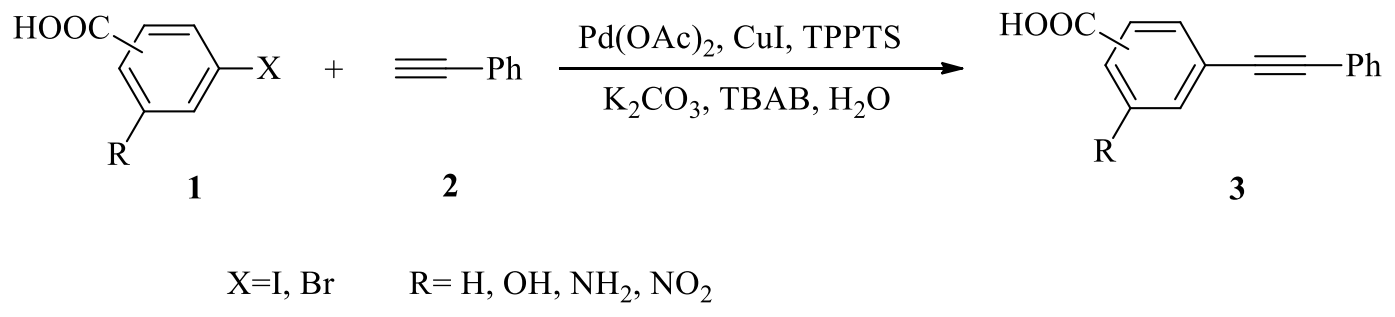

\begin{tabular}{cccc}
\hline Entry & Aryl halide & Product & Yield $(\%)^{\mathrm{b}}$ \\
\hline 1 & $4-\mathrm{IC}_{6} \mathrm{H}_{4} \mathrm{COOH}$ & $\mathbf{3 a}$ & $87(86)^{\mathrm{d}}$ \\
2 & $4-\mathrm{BrC}_{6} \mathrm{H}_{4} \mathrm{COOH}$ & $\mathbf{3 a}$ & $82(70)^{\mathrm{d}}$ \\
3 & $3-\mathrm{IC}_{6} \mathrm{H}_{4} \mathrm{COOH}$ & $\mathbf{3 b}$ & 85 \\
4 & $3-\mathrm{BrC}_{6} \mathrm{H}_{4} \mathrm{COOH}$ & $\mathbf{3 b}$ & 78 \\
$5^{\mathrm{c}}$ & $3-\mathrm{OH}-5-\mathrm{IC}_{6} \mathrm{H}_{3} \mathrm{COOH}$ & $\mathbf{3 c}$ & 80 \\
$6^{\mathrm{c}}$ & $3-\mathrm{OH}-5-\mathrm{BrC}_{6} \mathrm{H}_{3} \mathrm{COOH}$ & $\mathbf{3 c}$ & 73 \\
7 & $3-\mathrm{NH}{ }_{2}-5-\mathrm{IC}_{6} \mathrm{H}_{3} \mathrm{COOH}$ & $\mathbf{3 d}$ & 81 \\
8 & $3-\mathrm{NH}_{2}-5-\mathrm{BrC}_{6} \mathrm{H}_{3} \mathrm{COOH}$ & $\mathbf{3 d}$ & 76 \\
9 & $3-\mathrm{I}-5-\mathrm{NO}_{2} \mathrm{C}_{6} \mathrm{H}_{3} \mathrm{COOH}$ & $\mathbf{3 e}$ & 90 \\
10 & $3-\mathrm{Br}_{-5}-\mathrm{NO}_{2} \mathrm{C}_{6} \mathrm{H}_{3} \mathrm{COOH}$ & $\mathbf{3 e}$ & 85 \\
\hline
\end{tabular}

${ }^{\text {a }}$ Reaction conditions: 1 (10 mmol), 2 (15 mmol), Pd(OAc) $)_{2}(2 \mathrm{~mol} \%)$, TPPTS (8 mol\%), CuI (2 $\mathrm{mol} \%), \mathrm{TBAB}(6 \mathrm{~mol} \%), \mathrm{K}_{2} \mathrm{CO}_{3}(20 \mathrm{mmol})$ and $\mathrm{H}_{2} \mathrm{O}(20 \mathrm{ml})$ at $100{ }^{\circ} \mathrm{C}$ for $12 \mathrm{~h}$.

${ }^{\mathrm{b}}$ Average isolated yield from two independent trials.

${ }^{\mathrm{c}}$ The molar ratio of the substrates to $\mathrm{K}_{2} \mathrm{CO}_{3}$ was $1: 3$.

${ }^{\mathrm{d}}$ At $80{ }^{\circ} \mathrm{C}$.

As previously, literature suggested, aryl iodides are more reactive than aryl bromides in Pd-Catalyzed Sonogashira coupling reactions. ${ }^{24}$ Results of our experiments were in accordance with the literature. Under the optimal conditions, the coupling reactions between aryl iodides and phenylacetylene produced higher yields than aryl bromides counterparts (Table 2, entry 1 vs. 2, 3 vs. 4,5 vs. 6,7 vs. 8,9 vs. 10). And when the reaction temperature dropped to $80{ }^{\circ} \mathrm{C}$, the coupling reaction between 4-iodobenzoic acid and phenylacetylene gave the desired product in $86 \%$ yield, while 4-bromobenzoic acid only gave a $70 \%$ yield (Table 2 , entry $1^{\mathrm{d}}$ vs. $2^{\mathrm{d}}$ ). Unfortunately, the coupling of aryl chlorides (such as p-chlorobenzoic acid) with phenylacetylene failed to provide the desired products. 
The effect of substituted groups was studied in this reaction. According to the results obtained, halogenated aromatic acids with electron-withdrawing group, such as 3-iodo-5-nitrobenzoic acid and 3-bromo-5-nitrobenzoic acid, gave excellent yields of $85 \%$ and $90 \%$, respectively (Table 2, entry 9 and 10). In contrast, halogenated aromatic acids with electron-donating groups, such as 3-hydroxy-5-iodobenzoic acid, 3-hydroxy-5-bromobenzoic acid, 3-amino-5-iodobenzoic acid and 3-amino-5-bromobenzoic acid, gave the coupling products in $80 \%, 73 \%, 81 \%$ and $76 \%$ yields, respectively (Table 2, entries 5-8). The results are in agreement with the past reports showing that aryl halides containing electron-withdrawing groups are more reactive than aryl halides containing electron-donating groups in Sonogashira coupling reactions. ${ }^{25-27}$ In addition, it was observed that there was no considerable difference in yields between para-substituted and meta-substituted halogenated aromatic acids (Table 2, entry 3 vs. 1 and 4 vs. 2).

In order to show the advantage of using water compared with organic solvents, we have studied the reaction of 4-bromobenzoic acid as a model compound with phenylacetylene using $\mathrm{Pd}\left(\mathrm{PPh}_{3}\right) \mathrm{Cl}_{2}-\mathrm{CuI} / \mathrm{PPh}_{3}$ system in THF/Et ${ }_{3} \mathrm{~N}^{10}$ and also $\mathrm{Pd}(\mathrm{OAc})_{2}-\mathrm{CuI} / \mathrm{TPPTS}$ system in water. As we have observed, using $\mathrm{Pd}(\mathrm{OAc})_{2}-\mathrm{CuI} / \mathrm{TPPTS}$ system in water for this reaction afforded the product in a higher yield than using $\mathrm{Pd}\left(\mathrm{PPh}_{3}\right) \mathrm{Cl}_{2}-\mathrm{CuI} / \mathrm{PPh}_{3}$ system in $\mathrm{THF} / \mathrm{Et}_{3} \mathrm{~N}$. It showed that this method was more effective and green for the coupling reactions between halogenated aromatic acids and phenylacetylene.

The results suggested that phenylacetylene was effective for this reaction. Under the simple and environmentally benign reaction conditions, a series of substituted alkynes bearing carboxylic group were synthesized via one-step Sonogashira coupling between phenylacetylene and halogenated aromatic acids in good to excellent yields.

\section{Conclusions}

In summary, we have developed an effective, economic and green reaction condition for protecting-group-free synthesis of carboxylic group substituted alkynes for the first time. Moreover, a variety of important functional groups could be tolerated under the reaction conditions. In comparison to conventional synthetic methods, the novel method has wide potential prospects for the advantages of green economics, convenient manipulations and high yields, which makes it an attractive alternative for the cross-coupling reaction of halogenated aromatic acids with phenylacetylene.

\section{Experimental Section}

General. All reactions and manipulations were run under argon atmosphere. Melting points were determined on XRC melting point apparatus and uncorrected. ${ }^{1} \mathrm{H}$ NMR $(400 \mathrm{MHz})$ and ${ }^{13} \mathrm{C}$ 
NMR (100 MHz) spectra were recorded on a Perkin-Elmer R32 spectrometer in DMSO- $d_{6}$ or $\mathrm{CDCl}_{3}$ solvents with TMS as an internal standard. FTIR spectra were recorded on a PK1600 FTIR-type spectrophotometer using $\mathrm{KBr}$ pellets. Elemental analysis was recorded on Euro EA 3000 apparatus. Chemicals were obtained from commercial sources as analytical reagents and were, in general, used without further purification.

\section{General procedure for the Sonogashira coupling reaction}

To a stirred solution of halogenated aromatic acid $(10 \mathrm{mmol})$ and $\mathrm{K}_{2} \mathrm{CO}_{3}(20 \mathrm{mmol})$ in $20 \mathrm{ml}$ $\mathrm{H}_{2} \mathrm{O}$ were added $\mathrm{Pd}(\mathrm{OAc})_{2}(0.045 \mathrm{~g}, 0.20 \mathrm{mmol})$, TPPTS $(0.46 \mathrm{~g}, 0.80 \mathrm{mmol})$, TBAB $(0.19 \mathrm{~g}$, $0.60 \mathrm{mmol})$ and $\mathrm{CuI}(0.038 \mathrm{~g}, 0.20 \mathrm{mmol})$ under argon atmosphere. After stirred at $40{ }^{\circ} \mathrm{C}$ for 20 min, phenylacetylene $(15 \mathrm{mmol})$ was added, and the mixture was heated at $100{ }^{\circ} \mathrm{C}$ for $8-16 \mathrm{~h}$. Then the mixture was neutralized by concentrated $\mathrm{HCl}$ to $\mathrm{pH}=2-3$ and extracted with EtOAc $(3 \times 10 \mathrm{~mL})$. The organic layer was washed with water, dried on $\mathrm{MgSO}_{4}$ and after filtration, the solvent was removed. Further purification was performed by column chromatography (EtOAc/MeOH) to obtain the desired coupling product.

4-(Phenylethynyl)benzoic acid (3a). White solid, mp 218-220 ${ }^{\circ} \mathrm{C}$ (lit. ${ }^{28} \mathrm{mp} 218-219{ }^{\circ} \mathrm{C}$ ). ${ }^{1} \mathrm{H}$ NMR (400 MHz, DMSO-d6, $\delta$ ppm): $13.18(\mathrm{~s}, 1 \mathrm{H}), 7.98(\mathrm{~d}, J=5.6,2 \mathrm{H}), 7.68(\mathrm{~d}, J=5.2,2 \mathrm{H})$, 7.60-7.61 (m, 2H), 7.46-7.47 (m, 3H). $\left.{ }^{13} \mathrm{C} \mathrm{NMR} \mathrm{(100} \mathrm{MHz,} \mathrm{CDCl}_{3}, \delta \mathrm{ppm}\right): 166.6,131.5,131.5$, $130.6,129.5,129.2,128.8,126.6,121.8,91.9,88.6$.

3-(Phenylethynyl)benzoic acid (3b). White solid, mp 160-162 ${ }^{\circ} \mathrm{C}$ (lit. ${ }^{29} \mathrm{mp} 160-161{ }^{\circ} \mathrm{C}$ ). ${ }^{1} \mathrm{H}$ NMR (400 MHz, DMSO- $d_{6}, \delta$ ppm): $13.28(\mathrm{~s}, 1 \mathrm{H}), 8.07$ (s, 1H), 7.97 (d, $\left.J=7.6,1 \mathrm{H}\right), 7.81$ (d, $J$ $=7.2,1 \mathrm{H}), 7.56-7.60(\mathrm{~m}, 3 \mathrm{H}), 7.45-7.46(\mathrm{~m}, 3 \mathrm{H}) .{ }^{13} \mathrm{C}$ NMR $\left(100 \mathrm{MHz}, \mathrm{DMSO}-d_{6}, \delta \mathrm{ppm}\right)$ : 167.0, 135.8, 132.4, 132.0, 129.9, 129.7, 129.5, 129.2, 123.2, 122.4, 90.5, 88.8.

3-Hydroxy-5-(phenylethynyl)benzoic acid (3c). Brown solid, mp 198-200 ${ }^{\circ} \mathrm{C} .{ }^{1} \mathrm{H}$ NMR (400 MHz, DMSO- $d_{6}, \delta$ ppm): $13.14(\mathrm{~s}, 1 \mathrm{H}), 10.17$ (s, 1H), 7.57-7.60 (m, 2H), $7.52(\mathrm{~s}, 1 \mathrm{H}), 7.43-7.45$ $(\mathrm{m}, 3 \mathrm{H}), 7.38(\mathrm{~s}, 1 \mathrm{H}), 7.14(\mathrm{~s}, 1 \mathrm{H}) .{ }^{13} \mathrm{C}$ NMR (100 MHz, DMSO- $\left.d_{6}, \delta \mathrm{ppm}\right): 166.5,157.6,132.7$, 131.4, 128.9, 128.7, 123.5, 123.0, 122.0, 121.7, 116.6, 114.6, 89.4, 88.4; IR (KBr, cm $\left.{ }^{-1}\right): 3287$ (O-H), $3085(\mathrm{C}-\mathrm{H}), 2849,2666(\mathrm{COO}-\mathrm{H}), 2214(\mathrm{C} \equiv \mathrm{C}), 1714(\mathrm{C}=\mathrm{O}), 1250(\mathrm{C}-\mathrm{O})$; Anal. Calcd for $\mathrm{C}_{15} \mathrm{H}_{10} \mathrm{O}_{3}$ : C, 75.62; H, 4.23. Found: C, 75.44; H, 4.25.

3-Amino-5-(phenylethynyl)benzoic acid (3d). Pale-brown solid, mp 176-178 ${ }^{\circ} \mathrm{C} .{ }^{1} \mathrm{H}$ NMR (400 MHz, DMSO- $d_{6}, \delta$ ppm): 7.51-7.53 (m, 2H), 7.40-7.44 (m, 3H), 7.23 (s, 1H), 7.18 (s, 1H), 6.66 $(\mathrm{s}, 1 \mathrm{H}), 5.10(\mathrm{~s}, 2 \mathrm{H}) .{ }^{13} \mathrm{C}$ NMR $\left(100 \mathrm{MHz}, \mathrm{DMSO}-d_{6}, \delta \mathrm{ppm}\right): 170.3(\mathrm{COOH}), 148.5,141.8$, 131.7, 129.2, 128.8, 123.3, 121.4, 121.2, 116.9, 116.7, 91.4, 87.7; IR (KBr, cm $\left.{ }^{-1}\right)$ : 3462, 3371 $(\mathrm{N}-\mathrm{H}), 2922,2852(\mathrm{COO}-\mathrm{H}), 2202(\mathrm{C} \equiv \mathrm{C}), 1698(\mathrm{C}=\mathrm{O}), 1250(\mathrm{C}-\mathrm{O})$; Anal. Calcd for $\mathrm{C}_{15} \mathrm{H}_{11} \mathrm{NO}_{2}$ : C, 75.94; H, 4.67; N, 5.90. Found: C, 75.82; H, 4.70; N, 5.92.

3-Nitro-5-(phenylethynyl)benzoic acid (3e). Yellow solid, mp 208-210 ${ }^{\circ} \mathrm{C} .{ }^{1} \mathrm{H}$ NMR (400 MHz, DMSO- $\left.d_{6}, \delta \mathrm{ppm}\right): 14.00(\mathrm{~s}, 1 \mathrm{H}), 8.58(\mathrm{~s}, 2 \mathrm{H}), 8.41(\mathrm{~s}, 1 \mathrm{H}), 7.66-7.68(\mathrm{~m}, 2 \mathrm{H}), 7.48-7.49(\mathrm{~m}$, $3 \mathrm{H}) .{ }^{13} \mathrm{C}$ NMR (100 MHz, DMSO- $\left.d_{6}, \delta \mathrm{ppm}\right): 165.3,148.6,137.7,133.6,132.2,130.1,129.8$, 129.3, 125.0, 123.8, 121.7, 92.8, 86.8; IR (KBr, cm $\left.{ }^{-1}\right)$ : 3094 (C-H), 2543, 2845 (COO-H), 2214 
$(\mathrm{C} \equiv \mathrm{C}), 1698(\mathrm{C}=\mathrm{O}), 1535,1352(\mathrm{~N}-\mathrm{O}), 1286(\mathrm{C}-\mathrm{O})$; Anal. Calcd for $\mathrm{C}_{15} \mathrm{H}_{9} \mathrm{NO}_{4}$ : C, 67.42; H, 3.39; N, 5.24. Found: C, 67.29; H, 3.42; N, 5.26.

\section{Acknowledgements}

The authors are grateful to the National Undergraduates' Innovative Experiment Project of China and the Undergraduates' Innovative Experiment Project of Sichuan University for financial support.

\section{References}

1. Negishi, E.; Anastasia, L. Chem. Rev. 2003, 103, 1979.

2. Kobayashi, K.; Kobayashi, N.;Ikuta, M.; Therrien, B.; Sakamoto, S.; Yamaguchi, K. J. Org. Chem. 2005, 70, 749.

3. Maeda, T.; Furusho, Y.; Sakurai, S. I.; Kumaki, J.; Okoshi, K.; Yashima, E. J. Am. Chem. Soc. 2008, 130, 7938.

4. Bérubé, M.; Poirier, D. Org. Lett. 2004, 6, 3127.

5. Zeng, D.; Cheng, J.; Ren, S.; Sun, J.; Zhong, H.; Xu, E.; Du, J.; Fang, Q. React. Funct. Polym. 2008, 68, 1715.

6. Johnson, A. T.; Wang, L.; Standeven, A. M.; Escobar, M.; Chandraratna, R. A. S. Bioorg. Med. Chem. 1999, 7, 1321.

7. Urazoe, D.; Mori, H.; Yamakawa, K. U.S. Patent 20050215 820. 2005.

8. Johnson, A. T.; Wang, L.; Gillett, S. J.; Chandraratna, R. A. S. Bioorg. Med. Chem. Lett. 1999, 9, 573.

9. Hasaninejad, A.; Zare, A.; Sharghi, H.; Shekouhy, M. Arkivoc. 2008, (xi), 64.

10. Wu, D. F.; Yang, M. J.; Wang, Y.; Gao, G. W.; Men, J. Chin. Chem. Lett. 2011, 22, 159.

11. López-Deber, M.; Castedo, L.; Granja, J. R. Org. Lett. 2001, 3, 2823.

12. Gu, Z.; Li, Z.; Liu, Z.; Wang, Y.; Liu, C.; Xiang, J. Catal. Commun. 2008, 9, 2154.

13. Anderson, K. W.; Buchwald, S. L. Angew. Chem. Int. Ed. 2005, 44, 6173.

14. Guan, J. T.; Weng, T. Q.; Yu, G. A.; Liu, S. H. Tetrahedron Lett. 2007, 48, 7129.

15. Lipshutz, B. H.; Chung, D. W.; Rich, B. Org. Lett. 2008, 10, 3793.

16. Ye, Z. W.; Yi, W. B. J. Fluorine. Chem. 2008, 129, 1124.

17. Liang, B.; Dai, M.; Chen, J.; Yang, Z. J. Org. Chem. 2005, 70, 391.

18. Pschierer, J.; Plenio, H. Org. Lett. 2010, 12, 5358.

19. Firouzabadi, H.; Iranpoor, N.; Gholinejad, M. J. Mol. Catal. A. Chem. 2010, 321, 110.

20. Raju, S; Kumar, P. R; Mukkanti, K.; Annamalai, P.; Pal, M. Bioorg. Med. Chem. Lett. 2006, $16,6185$.

21. Suzuka, T.; Okada, Y.; Ooshiro, K.; Uozumi, Y. Tetrahedron. 2010, 66, 1064. 
22. Bakherad, M.; Keivanloo, A.; Kalantar, Z.; Jajarmi, S. Tetrahedron Lett. 2011, 52, 228.

23. Genet, J. P.; Savignac, M. J. Organomet. Chem. 1999, 576, 305.

24. Hundertmark, T.; Littke, A. F.; Buchwald, S. L.; Fu, G. C. Org. Lett. 2000, 2, 1729.

25. Heravi, M. M.; Keivanloo, A.; Rahimizadeh, M.; Bakavoli, M.; Ghassemzadeh, M. Tetrahedron Lett. 2004, 45, 5747.

26. Cai, M.; Xu, Q.; Sha, J. J. Mol. Catal. A. Chem. 2007, 272, 293.

27. Islam, S. M.; Mondal, P.; Roy, A. S.; Mondal, S.; Hossain, D. Tetrahedron Lett. 2010, 51, 2067.

28. Wang, J. X.; Liu, Z. X.; Hu, Y. L.; Wei, B. G.; Kang, L. Q. Synth. Commun. 2002, 32, 1937.

29. Kochi, J. K.; Hammond, G. S. J. Am. Chem. Soc. 1953, 75, 3452. 